\title{
HD 17156 : a progress report
}

\author{
Mauro Barbieri ${ }^{1}$, Roi Alonso ${ }^{1}$, M. Cecconi ${ }^{3}$, R. U. Claudi ${ }^{2}$, S. \\ Desidera $^{2}$, M. Endl ${ }^{4}$, A. F. Martinez Fiorenzano ${ }^{3}$, and R. Gratton ${ }^{2}$ \\ ${ }^{1}$ LAM, 38 bd. Joliot-Curie, 133XX Marseille Cedex 13, France \\ ${ }^{2}$ INAF Osservatorio Astronomico di Padova, Vicolo dell' Osservatorio 5, I-35122, Padova, Italy \\ ${ }^{3}$ INAF Fundacion Galileo Galilei, Santa Crux de La Palma, Spain \\ ${ }^{4}$ McDonald Observatory, The University of Texas at Austin, Austin, TX 78712, USA
}

\begin{abstract}
We present an analysis of the HD 17156 planetary system based on a photometric transit dataset and radial velocities obtained on 3 December 2007. We also present limits on the presence of close stellar companions based on high resolution images.
\end{abstract}

\section{Introduction}

To date the family of transiting extrasolar planets is mainly composed of short-period planets, one notable exception is HD 17156b. This planet discovered by Fischer et al. (2007) was detected in transit by Barbieri et al. (2007). After the discovery of the transit we have conducted follow-up observations, radial velocity, photometric and highresolution imaging, for the characterization of the system. Here we present the results of these measurements.

\section{Photometric observations}

Photometric observations of the transit were obtained on 3 December 2007, with various telescopes. We have used two medium class telescopes (Asiago $1.82 \mathrm{~m}$ and OHP 1.20 $\mathrm{m}$ ) and some small telescopes (six $0.3 \mathrm{~m}$ telescopes operated by amateurs) all located in continental Europe, and the Telast $0.3 \mathrm{~m}$ in the Canary Islands. During the night the weather conditions over Europe was not optimal due to the presence of a depressionary area over the British Isles, and the full transit was observed with six telescopes, the remaing three observatory obtained data only for the ingress phase of the transit. All the observations was obtained in $\mathrm{R}$ band. After standard reduction of the images, we performed aperture photometry of HD 17156 and several surrounding stars. The sum of the flux of the other stars was used as reference flux, and the lightcurve of HD 17156 was obtained by dividing its flux by the reference flux. We combined all the lightcurves of HD 17156 using temporal bins of 150 seconds, and the errors were evaluated as the standard rms over 30 minutes (the timescale of transit ingress/egress phase). Each ligthcurve and the combined one are showed in Fig. 1. Finally we performed the fit on the combined lightcurve using the formulation of Giménez (2006). The results of our best fit are summarized in Table 1.

\section{Radial velocity observations}

We observed HD 17156 on the night 3 Dec 2007, including continuous monitoring during the transit, with SARG, the high-resolution spectrograph of the TNG. Exposure 

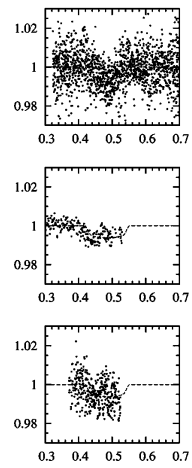
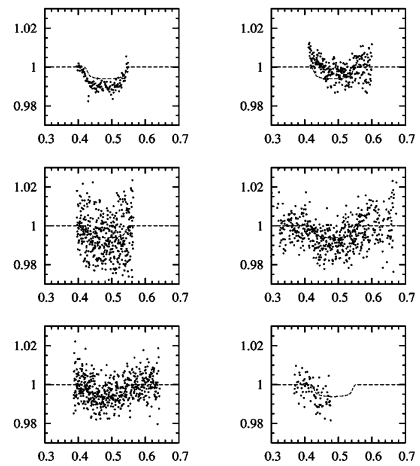

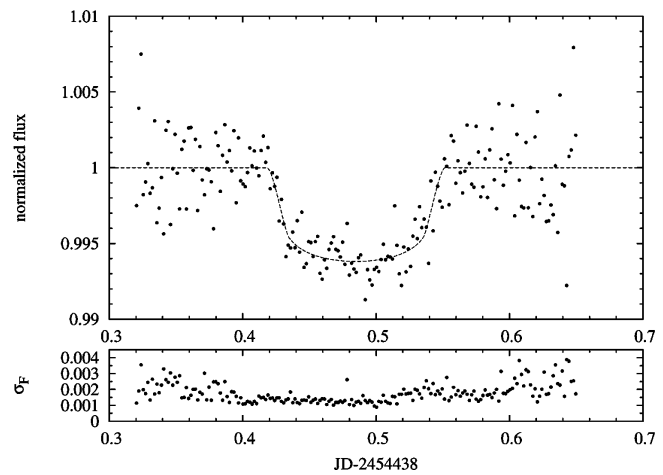

Figure 1. Left Mosaic of the differential light curves of the December 3/4 transit of HD 17156 from the various telescopes used. In each box the horizontal axis is $H J D-2454438$ and the vertical axis is the relative flux. In clockwise order from top left the lightcurves from: Univ. Siena, Mt. Baldo, R. Papini, Obs. Cavezzo, C. Lopresti, M. Vallerani, TELAST, ASIAGO, OHP. The best fit model is also shown. Right Composite light curve created from the nine light curves with the best fit model indicated by the solid line. Errors and residuals with respect to the best -it model are also displayed in the bottom panels.

Table 1. Parameters of the transit of HD 17156b

\begin{tabular}{|c|c|c|}
\hline $\begin{array}{l}\mathrm{k}_{r r}=R_{p} / R_{s} \\
\theta_{1}\end{array}$ & $\begin{aligned} 0.073 & \pm 0.003 \\
-0.0034 & \pm 0.0002\end{aligned}$ & \\
\hline i & $87.7 \pm 2.5$ & deg \\
\hline $\mathrm{T}_{c}$ & $2454438.4846 \pm 0.001$ & HJD \\
\hline
\end{tabular}

time of the spectra acquired with the iodine cell was $900 \mathrm{~s}$, typically resulting in a signalto-noise ratio of about 80 per pixel. One additional spectrum was obtained on 25 Oct 2007. These spectra were reduced and analyzed in the manner used for the planet search program on-going using SARG (Desidera et al. 2007) using the AUSTRAL code (Endl et al. 2000). Typical errors on individual measurements are $4 \mathrm{~m} / \mathrm{s}$. We used the SARG data together with the published RV values (Fischer et al. 2007; Narita et al. 2007) to derive the planetary orbit. For the fit we used only the out-of-transit measurements in our dataset and that of Narita et al. We use a downhill simplex algorithm to perform the RV fit of all the datasets including the zero-point shifts between the datasets as free parameters. A stellar jiitter of $3 \mathrm{~m} / \mathrm{s}$ was added in quadrature to the observational errors. The best fit results and relative uncertainty are presented in Table 2, and in Fig. 2 are show the phased radial velocity curve with the best fit model. We have performed the fit for the Rossiter-McLaughlin effect on RV measurements during the transit. We have used the Giménez (2006) analytical description, in order to minimize the number of free parameters, instead of fitting all parameters we have fixed all to the previous best fit values except $v \sin i$ and $\lambda$. We obtained $v \sin i=1.9 \pm 0.5 \mathrm{~km} / \mathrm{s}$ and $\lambda=6.5 \pm 5^{\circ}$, and the best fit solution is portraied in Fig. 2. Our results show that in this planetary system there is a spin-orbit alignment, and we do not confirm the tentative detection of misalignement of Narita et al. (2007) †.

$\dagger$ After the IAU Symposium, Cochran et al. (2008) have published independent RV measurements of HD 17156 obtained during the transit of 25 December 2007. They have used these data for the measurements of the RM effect, without finding any significant misalignement in the system. 

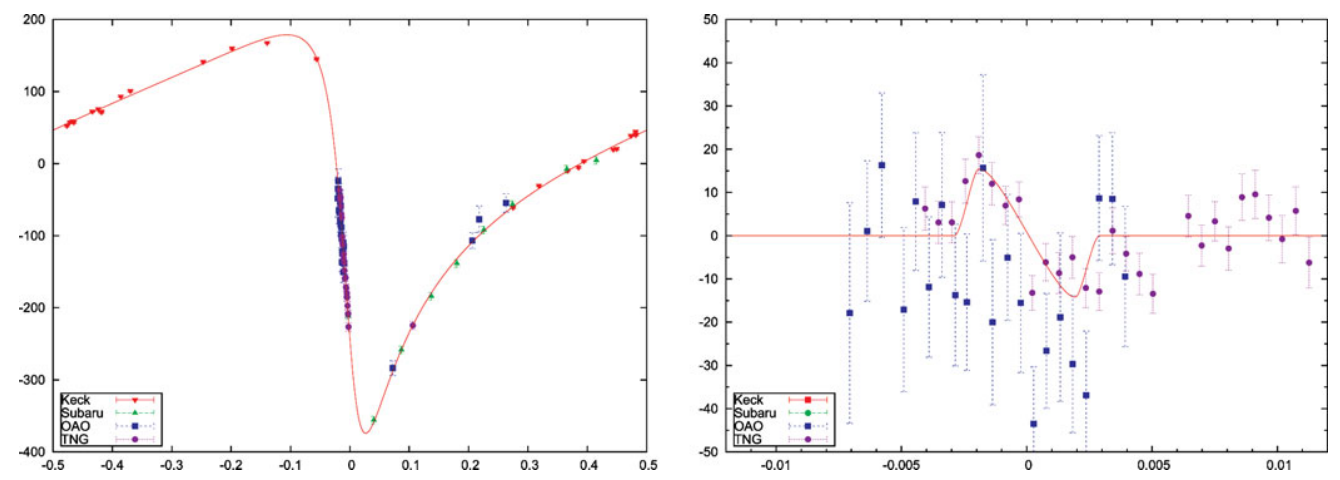

Figure 2. Left Radial velocities of HD 17156 phased to the best-fit orbital solution. Right Orbital radial velocity residuals of HD 17156 during the transit, and best-fit model of the Rossiter-McLaughlin effect.

Table 2. Orbital and RM best-fit parameters of HD 17156b from radial velocities.

\begin{tabular}{lcl}
\hline $\mathrm{K}$ & $277.4 \pm 4.1$ & $\mathrm{~m} / \mathrm{s}$ \\
$\mathrm{P}$ & $21.2178 \pm 0.0011$ & day \\
$\mathrm{e}$ & $0.678 \pm 0.007$ & \\
$\omega$ & $121.5 \pm 1.0$ & $\mathrm{deg}$ \\
$\mathrm{T}_{P}$ & $2454438.7708 \pm 0.0090$ & $\mathrm{HJD}$ \\
shift Keck & $93.4 \pm 0.7$ & $\mathrm{~m} / \mathrm{s}$ \\
shift Subaru & $95.5 \pm 4.0$ & $\mathrm{~m} / \mathrm{s}$ \\
shift OAO & $141.5 \pm 8.0$ & $\mathrm{~m} / \mathrm{s}$ \\
shift SARG & $132.4 \pm 2.3$ & $\mathrm{~m} / \mathrm{s}$ \\
\hline$\lambda$ & $6.5 \pm 5.0$ & $\mathrm{deg}$ \\
$v \sin i$ & $1.9 \pm 0.5$ & $\mathrm{~km} / \mathrm{s}$ \\
\hline
\end{tabular}

\section{Adaptive optics observations}

HD 17156 was observed with AdOpt@TNG, the adaptive optics module of TNG. The instrument feeds the HgCdTe Hawaii 1024x1024 detector of NICS, the near infrared camera and spectrograph of TNG, providing a field of view of about $44 \times 44$ arcsec, with a pixel scale of $0.0437 \mathrm{arcsec} /$ pixel. Series of $15 \mathrm{sec}$ images on HD 17156 were acquired on 3, 18 and 23 October 2007 in $\operatorname{Br} \gamma$ intermediate-band filter. Images were taken moving the target in different positions on the detector, to allow sky subtraction without the need for additional observations, and each night at three different field orientations to make it easier disentagling true companions from image artefacts. The target itself was used as guide star for the adaptive optics. The subsequent analysis was optimized for the detection of companions in different separation ranges. At small separation we use the two best combined images taken at different field orientations on Oct 18, at separation larger than 2 arcsec we summed all the images properly rotated, obtaining a deep image over a field of about $10 \times 10$ arcsec (Fig. 3). No true companions are seen in both the differential image at small separation and the deep combined image. The limit for detection was fixed at peak intensities 5 times the dispersion over annuli at different radial separation. The results, both for the differential image and the deep composite image, are shown in Fig. 4 using a mass-luminosity relation for low-mass stars and converting projected separation in arcsec to AU using the Hipparcos distance to the star. The result is that main-sequence stars can be excluded from about 150 to 1000 AU. At such separation only brown dwarf or white dwarf companions are compatible with our detection limits. 

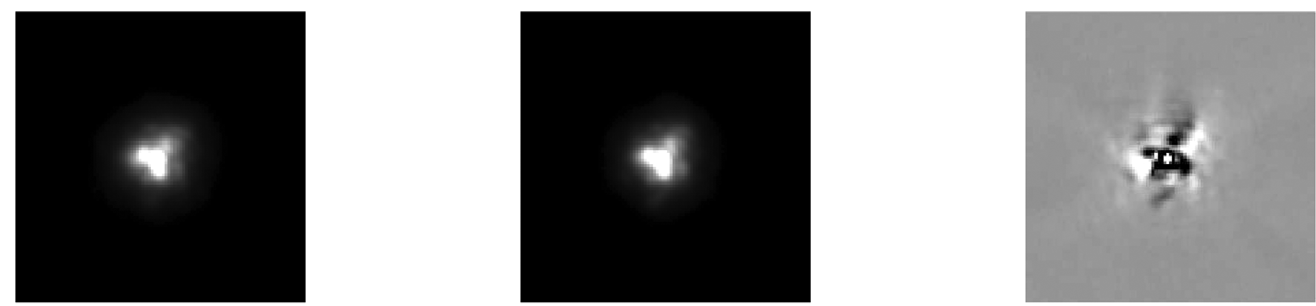

Figure 3. Images of HD 17156. Left and central panels; images taken with two different field orientations. A similar quasi-static speckle pattern can be seen. Right panel: difference between the two previous images, that allow to improve significantly detection limits in the inner regions. The field of view shown in figure is $4.3 \times 4.3$ arcsec. The images are displayed in linear gray scale.
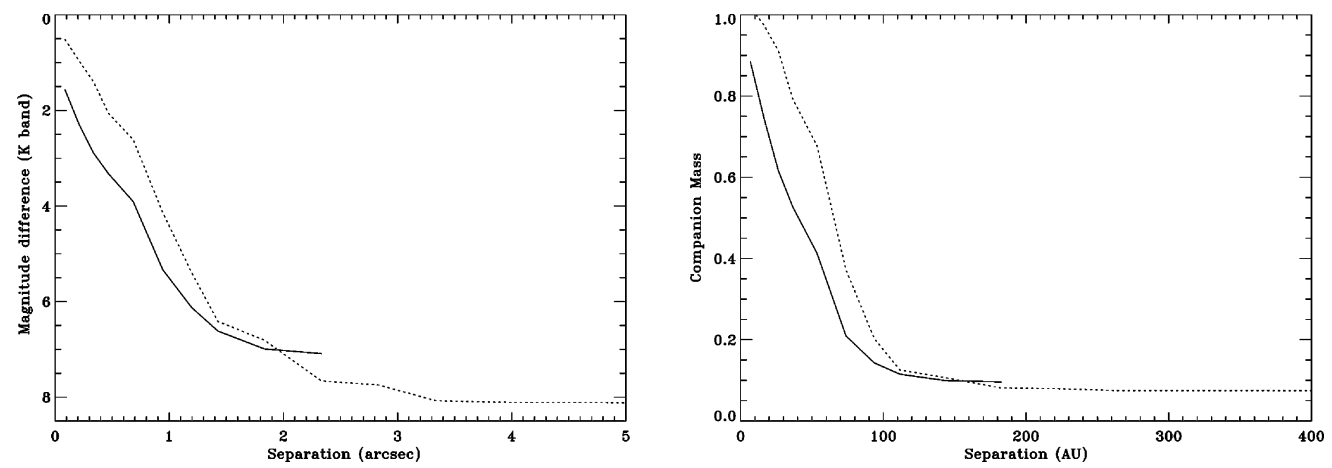

Figure 4. Left panel Detectability limits for companions of different magnitude around HD 17156 as a function of the projected separation in arcsec. Continuous line: limits on the difference image. Dotted line: limits on the composite deep image. Right panel Detectability limits for stellar companions around HD 17156 as a function of the projected separation in AU. Continuous line: limits on the difference image. Dotted line: limits on the composite deep image.

At smaller separation detectability gets increasingly worse, and only stars with mass larger than about $0.4 \mathrm{M}_{\odot}$ can be excluded at a projected separation of $50 \mathrm{AU}$.

\section{Acknowledgements}

Based on observations made with the Italian Telescopio Nazionale Galileo (TNG) operated on the island of La Palma by the Fundacion Galileo Galilei of the INAF (Istituto Nazionale di Astrofisica) at the Spanish Observatorio del Roque de los Muchachos of the Instituto de Astrofisica de Canarias.

\section{References}

Barbieri et al. 2007, A\&A, 476, 13

Cochran, W. D. et al. 2008, ArXiv e-prints, 806, arXiv:0806.4142

Desidera, S., et al. 2007, ArXiv e-prints, 705, arXiv:0705.3141

Endl, M., Kürster, M., Els, S. 2000, A\&A, 363, 585

Fischer, D. A., et al. 2007, ApJ, 669, 1336

Giménez, A. 2006, A\&A, 450, 1231

Giménez, A. 2006, ApJ, 650, 408

Narita, N., Sato, B., Ohshima, O., Winn, J. N. 2008, PASJ, 59, 763 